\title{
The reconstruction of an equation of visco-elasticity
}

https://doi.org/10.1515/msds-2018-0012

Received September 27, 2018; accepted December 13, 2018

Abstract: We are concerned with the reconstruction of two coefficients of an integro-differential equation modeling the deformation of materials with memory. We show that we can explicitly reconstruct the memory, the source terms and the diffusion constant from two observations only.

\section{Introduction}

Consider the inverse problem of recovering the function $h$, which models the memory effect, the control $p$, and the diffusion constant $k$, as treated by F. Colombo, D. Guidetti and V. Vespri, see [2],

$$
\left\{\begin{array}{l}
u_{t}(x, t)=k \Delta u(x, t)+\int_{0}^{t} h(t-s) \Delta u(x, s) d s+p(t) g(x) \quad x \in \Omega \subset \mathbb{R}^{d}, d \geq 2 \\
u(x, 0)=a(x) \\
\partial_{n} u(x, t)=0 \quad \text { where } x \in \partial \Omega
\end{array}\right.
$$

from the knowledge of two given observations

$$
G_{i}(t)=\int_{\Omega} u(x, t) \phi_{i}(x) d x \quad \text { for } t \geq 0 \text { and } i=1,2
$$

where $\phi_{i}$ is to be chosen appropriately later. We now can state the problem:

Problem: Given the functions $a, g \in L^{2}(\Omega)$ reconstruct $\{k, h(t), p(t)\}$ from two observations $G_{i}(t)$.

This note is a follow up of the work in [2] where global existence of the solution of the direct problem was shown, and so (2) holds. Since the uniqueness of the inverse problem of recovering $\{k, h(t), p(t)\}$ was proved to follow from two observations, see problem 1.2 in [2], it remains to find a reconstruction procedure which is the objective of this note. We shall do so with fewer and weaker conditions as our explicit procedure requires two simple $G_{i}$. As material science is a rapidly growing area, it calls for new and simple computational methods that helps design materials with new properties by getting explicit results from reverse engineering, [2-4].

\section{Preliminaries}

This note discusses the reconstruction aspects of the functions $\{k, h, p\}$ from two measurements only, see Problem 1.2 in [2]. The method is simple and makes use of the spectral theory of the Laplacian under Neumann boundary conditions which we recall now. Let us agree to denote by $\lambda_{n}$ and $\varphi_{n}$ its eigenvalues and eigenfunctions

$$
\left\{\begin{array}{l}
-\Delta \varphi_{n}(x)=\lambda_{n} \varphi_{n}(x) \quad x \in \Omega \\
\partial_{n} \varphi_{n}(x)=0 \quad \text { if } \quad x \in \partial \Omega
\end{array}\right.
$$

\footnotetext{
^Corresponding Author: Amin Boumenir: Department of Mathematics,UWG, Carrollton, USA, E-mail: boumenir@westga.edu
} 
Recall that $\lambda_{1}=0, \lambda_{n} \rightarrow \infty$ and that $\varphi_{n}$ is an eigenbasis of $L^{2}(\Omega)$, which we normalize by $\left\|\varphi_{n}\right\|=1$. We assume that the boundary $\partial \Omega$ is smooth and

$$
h, p \in L^{1}(0, \infty) \text { are unknown but we are given } a, g \in L^{2}(\Omega) .
$$

The fact that $g$ is known means that all its Fourier coefficients $g_{n}=\int_{\Omega} g(x) \varphi_{n}(x) d x$ are also given. We now prove the following lemma

Lemma 1. If $0 \leq g \neq$ constant in $L^{2}(\Omega)$ then at least two Fourier coefficients are non zero, $g_{1} \neq 0$ and $g_{n} \neq 0$ with $n \geq 2$.

Proof. Since $0 \leq g \neq 0$ then $g_{1}=|\Omega|^{-1 / 2} \int_{\Omega} g(x) d x>0$. Next $g(x)=\sum g_{n} \varphi_{n}(x)$ and if all $g_{n}=0$ for $n \geq 2$ then $g(x)=g_{1}|\Omega|^{-1}=$ constant which is impossible.

The unique solution of (1), which is shown to exist globally in [2], can be written as

$$
u(x, t)=\sum_{n \geq 1} c_{n}(t) \varphi_{n}(x)
$$

which converges in $L^{2}(\Omega)$ and

$$
c_{n}(t)=\int_{\Omega} u(x, t) \varphi_{n}(x) d x .
$$

In order to define the observation $G_{i}$, by (2), we shall choose $\phi_{i}$ so to filter and observe one $c_{i}$ at a time only from the solution (4). To this end multiply (1) by $\varphi_{n}$ and integrate to get a sequence of equations involving the Fourier coefficients $c_{n}$

$$
\int_{\Omega} u_{t}(x, t) \varphi_{n}(x) d x=k \int_{\Omega} \Delta u(x, t) \varphi_{n}(x) d x+\int_{\Omega} \int_{0}^{t} h(t-s) \Delta u(x, s) d s \varphi_{n}(x) d x+p(t) \int_{\Omega} g(x) \varphi_{n}(x) d x
$$

which reduces to the system

$$
c_{n}^{\prime}(t)=-\lambda_{n} k c_{n}(t)-\lambda_{n} \int_{0}^{t} h(t-s) c_{n}(s) d s+p(t) g_{n}
$$

where we recall that $c_{n}(0)=\int_{\Omega} a(x) \varphi_{n}(x) d x$ and $g_{n}=\int_{\Omega} g(x) \varphi_{n}(x) d x$ are known but $k, h$ and $p$ are still unknown.

We first start by finding the function $p$. To this end we use, $\phi_{1}(x):=\varphi_{1}(x)=|\Omega|^{-1 / 2}$, and (5), to obtain

$$
G_{1}(t)=|\Omega|^{-1 / 2} \int_{\Omega} u(x, t) d x=c_{1}(t) \text { and so } \quad G_{1}(0)=|\Omega|^{-1 / 2} \int_{\Omega} a(x) d x
$$

From (6) and the fact $\lambda_{1}=0$, we get

$$
c_{1}^{\prime}(t)=p(t) g_{1}
$$

and since $g_{1} \neq 0$, we get

$$
p(t)=\frac{1}{g_{1}} G_{1}^{\prime}(t)=\frac{1}{\int_{\Omega} g(x) d x} \frac{d}{d t} \int_{\Omega} u(x, t) d x .
$$

Going back to (6), use $g_{n} \neq 0$, and $t=0$, to get

$$
c_{n}^{\prime}(0)=-\lambda_{n} k c_{n}(0)+p(0) g_{n}
$$

and thus

$$
k=\left(p(0) g_{n}-c_{n}^{\prime}(0)\right) / \lambda_{n} c_{n}(0)=\left(p(0) g_{n}-G_{n}^{\prime}(0)\right) / \lambda_{n} G_{n}(0) .
$$

This means that we need to choose the corresponding $c_{n}(0) \neq 0$. However this is easily achieved by taking $a(x)=\sum_{n \geq 1} \varphi_{n}(x) / n \in L^{2}(\Omega)$. 
Finally to find $h$, where we need to recall the global existence of the solution $u$, [2], means that equation (6) holds for $t \in(0, \infty)$ and so we can use the Laplace transform, and with $h \in L^{1}(0, \infty)$, we get

$$
s \mathcal{L}\left(c_{n}\right)(s)=-\lambda_{n} k \mathcal{L}\left(c_{n}\right)(s)-\lambda_{n} \mathcal{L}(h)(s) \mathcal{L}\left(c_{n}\right)(s)+\mathcal{L}(p)(s) g_{n}+c_{n}(0) .
$$

and so

$$
\lambda_{n} \mathcal{L}(h)(s)=-s-\lambda_{n} k+\frac{\mathcal{L}(p)(s) g_{n}+c_{n}(0)}{\mathcal{L}\left(c_{n}\right)(s)}
$$

while we have from $(7), \mathcal{L}(p)(s)=\frac{s \mathcal{L}\left(G_{1}\right)(s)-G_{1}(0)}{g_{1}}$. It follows then

$$
\lambda_{n} \mathcal{L}(h)(s)=s\left(\frac{g_{n} \mathcal{L}\left(G_{1}\right)(s)-g_{1} \mathcal{L}\left(c_{n}\right)(s)}{g_{1} \mathcal{L}\left(c_{n}\right)(s)}\right)+\frac{g_{1} c_{n}(0)-g_{n} G_{1}(0)}{g_{1} \mathcal{L}\left(c_{n}\right)(s)}-\lambda_{n} k
$$

It remains to take the inverse Laplace transform to find $h$. Thus we have proved the main result of this note.

Proposition 2. Assume that $0 \leq g(x)=c$ in $L^{2}(\Omega)$, then we can reconstruct $\{k, h, p\}$ using two measurements $G_{i}$ for $i=1,2$ given by (2).

Remark: It is clear that the global existence of the solution $u$ means that $\mathcal{L}(h)$ is well defined. As for its inverse Laplace transform, either $\mathcal{L}(h)$ is found in tables or one has to use the many available inversion formulae to approximate $h$, see $[1,5]$.

Also it is known that from the physics point of view that $h(t) \leq 0$. This fact can easily be verified by Bernstein's theorem, [6, theorem 12f], that it is necessary and sufficient,

$$
(-1)^{n} \frac{d^{n}}{d s^{n}} \mathcal{L}(h)(s)=\int_{0}^{\infty} e^{-s t} t^{n} h(t) d t \leq 0 \quad \text { for any } n \geq 0 \text {, and } s>0 .
$$

Acknowledgement: The author sincerely thanks the referee for the valuable comments.

\section{References}

[1] A. Boumenir, and A. Al-Shuaibi, The inverse Laplace transform and analytic pseudo-differential operators. J. Math. Anal. Appl. 228 (1998), no. 1, 16 ?36.

[2] F. Colombo, D. Guidetti, and V. Vespri, Some global in time results for integro-differential parabolic inverse problems. Differential equations: inverse and direct problems, 35-58, Lect. Notes Pure Appl. Math., 251, Chapman Hall CRC, Boca Raton, FL, 2006.

[3] F. Colombo, D. Guidetti and V. Vespri, Identification of two memory kernels and the time dependence of the heat source for a parabolic conserved phase-field model. Math. Methods Appl. Sci. 28 (2005), no. 17, 2085-2115.

[4] F. Colombo and V. Vespri, A semi-linear integro-differential inverse problem, Evolution equations, J. Goldstein, R. Nagel and S. Romanelli editors, Marcel Dekker, Inc., Cap 6, 234 (2003), 91-104.

[5] V.I. Krylov, and N.S. Skoblya A Handbook of Methods of Approximate Fourier Transformation and Inversion of the Laplace Transform, Mir, Moscow (1977)

[6] D.V. Widder, The Laplace Transform, Dover, 2010 